\title{
Berufliche Allergien gegen Pepsin, Chymosin und mikrobielle Labersatzstoffe ${ }^{1}$
}

\section{Occupational Allergies against Pepsin, Chymosin and Microbial Rennet}

Autoren

Institute
V. van Kampen', H. Lessmann², T. Brüning ${ }^{1}$, R. Merget ${ }^{1}$

${ }^{1}$ Institut für Prävention und Arbeitsmedizin der Deutschen Gesetzlichen Unfallversicherung,

Institut der Ruhr-Universität Bochum (IPA) (Leiter: T. Brüning)

${ }^{2}$ Informationsverbund Dermatologischer Kliniken (IVDK) an der Universität Göttingen (Leiter: A. Schnuch) eingereicht 21.2.2013

akzeptiert 22. 2. 2013

Bibliografie

DOI http://dx.doi.org/

10.1055/s-0032-1326407

Online-Publikation: 20.3.2013

Pneumologie 2013; 67: 260-264

(c) Georg Thieme Verlag KG

Stuttgart · New York

ISSN 0934-8387

Korrespondenzadresse Dr. rer. nat. Vera van Kampen Institut für Prävention und Arbeitsmedizin der Deutschen Gesetzlichen Unfallversicherung, Institut der Ruhr-Universität Bochum (IPA)

Bürkle-de-la-Camp-Platz 1 44789 Bochum

kampen@ipa-dguv.de

Serienherausgeber

R. Merget, Bochum

D. Nowak, München

\section{Zusammenfassung}

\section{$\nabla$}

Lab ist ein Gemisch aus den proteinspaltenden Verdauungsenzymen Pepsin und Chymosin (Rennin), welches gewöhnlich aus dem Labmagen junger Wiederkäuer gewonnen wird. Während Pepsin auch in der Arzneimittelindustrie Einsatz findet, werden Pepsin und Chymosin gemeinsam zum Ausfällen des Milcheiweißes bei der Herstellung von Käse benötigt. Davon abzugrenzen ist das mikrobielle Lab, das natürlicherweise von bestimmten Mikroorganismen gebildet wird. Dieses wird als Lab-Austauschstoff bereits seit Jahrzehnten in der Käseherstellung verwendet. Schon seit längerem ist bekannt, dass eine berufliche Exposition gegenüber Enzymstäuben zu einer Soforttypallergie führen kann. Die vorliegende Arbeit gibt einen Überblick über die Ergebnisse einer Literaturauswertung hinsichtlich der berufsbedingten atemwegssensibilisierenden Wirkung von verschiedenen Lab-Enzymen. Da es an den verschiedenen Arbeitsplätzen nicht selten zu einer gleichzeitigen Exposition gegenüber Pepsin und Chymosin bzw. mikrobiellen Labersatzstoffen kommt, wurde die entsprechende Literatur gemeinsam ausgewertet. Durch die dargestellten Studien sind Fälle von spezifischer Überempfindlichkeit der Atemwege oder der Lunge gut belegt. Durch positive Ergebnisse im Haut- und Provokationstest sowie dem Nachweis spezifischer IgEAntikörper scheint ein immunologischer Wirkmechanismus gesichert.

\section{Abstract \\ $\nabla$}

Rennet is a mixture of the proteolytic enzymes pepsin and chymosin (rennin), which is usually obtained from the fourth stomach of young ruminants. While pepsin is also used in the pharmaceutical industry, both enzymes (pepsin and chymosin) are used for the coagulation of milk protein in the manufacture of cheese. Additionally, microbial rennet, which is naturally produced by certain microorganisms, has been used as a substitute for natural rennet in the cheese production for decades. Exposure to enzyme dusts has long been known to cause occupational immediate hypersensitivities. The present paper reviews the results of an evaluation of the literature data concerning occupational airway sensitisation due to natural and microbial rennet. Cases of specific airway sensitisation caused by rennet could be shown clearly by several studies. Positive skin prick and challenge tests as well as specific IgE antibodies have been described, thus suggesting an immunological mechanism.

\footnotetext{
${ }^{1}$ Diese Arbeit ist eine modifizierte Version zweier Publikationen der Senatskommission der Deutschen Forschungsgemeinschaft (DFG) zur Prüfung gesundheitsschädlicher Arbeitsstoffe (Pepsin: erschienen in der 47. Mitteilung der Toxikologisch-arbeitsmedizinischen
}

Begründungen von MAK-Werten, Wiley-VCH Verlag GmbH \& Co. KGaA, 2011; Mikrobielle Labersatzstoffe: erschienen in der 48. Mitteilung der Toxikologisch-arbeitsmedizinischen Begründungen von MAK-Werten, WileyVCH Verlag GmbH \& Co. KGaA, 2012). 


\section{Einleitung}

Bei Pepsin und dem verwandten Chymosin (Rennin) handelt es sich um proteinspaltende Verdauungsenzyme. Da sich bei beiden Enzymen Asparaginsäure als funktionelle Aminosäure im aktiven Zentrum befindet, zählen sie zu den Aspartatproteasen (Aspartatendopeptidasen).

In der EC-Nomenklatur zur Klassifikation von Enzymen wird Pepsin unter der Nummer EC 3.4.23.1 geführt. Es handelt sich um eine saure Endopeptidase mit einem Molekulargewicht von $36 \mathrm{kDa}$. Gebildet wird sie unter Einwirkung der Salzsäure des Magens aus einer inaktiven Vorstufe, dem Pepsinogen. Pepsin vermag Peptidbindungen vorrangig C-terminal zu den Aminosäuren Phenylalanin, Leucin und Glutaminsäure zu spalten. Die höchste Aktivität hat Pepsin bei einem pH-Wert zwischen 1,5 und 3; pH-Werte oberhalb von 6 inaktivieren das Enzym irreversibel. Temperaturen bis $60^{\circ} \mathrm{C}$ können seine Funktion nicht beeinträchtigen, ebenso wenig höhere Konzentrationen an Harnstoff und Guanidin. Pepsin wird in unterschiedlicher Form (PepsinWein, Tabletten) als Arzneimittel, insbesondere bei Verdauungsstörungen, eingesetzt. Darüber hinaus findet Pepsin Anwendung bei der künstlichen Verdauung von Fleischproben im Rahmen der Trichinenuntersuchung, die Teil der amtlichen Schlachttierund Fleischuntersuchung ist.

Während Pepsin vermutlich im Magen aller Säuger zu finden ist, geht man davon aus, dass Chymosin (EC 3.4.23.4.) nur in den Mägen der milchsaugenden Jungtiere vorkommt, da es für die Aufschließung des Milcheiweißes verantwortlich ist. Pepsin wird i.d.R. aus Schweine- oder Rindermägen isoliert und die kommerziell verfügbare Pepsin-Form ist meistens Pepsin A aus dem Magen des Schweins. Rinder-Chymosin findet sich als Labferment im vierten Magen (Labmagen) von Kälbern. ChymosinZubereitungen aus diesem Labferment enthalten stets auch Pepsin. Chymosin (oder ein entsprechender Austauschstoff, s. u.) ist für die Käseherstellung unverzichtbar, und auch Pepsin wird, meist im Gemisch mit Chymosin, zur Casein-Fällung bei der Käseherstellung benutzt.

Davon abzugrenzen ist das „mikrobielle Lab“, das als Lab-Austauschstoff bereits seit einigen Jahrzehnten bei der Käsezubereitung eingesetzt wird. Hierunter versteht man Aspartatproteasen, die natürlicherweise von bestimmten Mikroorganismen gebildet werden. So wurde ein Labersatz aus dem Schlauchpilz Endothia parasitica (heutiger Name: Cryphonectria parasitica) bereits seit 1967 in den USA unter der Handelsbezeichnung Suparen ${ }^{\circledR}$ verkauft [1]. Nach heutiger Nomenklatur handelt es sich hierbei um das relativ thermolabile, nicht glycosylierte Enzym Endothiapepsin (EC 3.4.23.24). Aus Kulturen der Zygomyceten Rhizomucor miehei (früher: Mucor miehei) oder Rhizomucor pusillus werden glycosylierte Mucoraspartatproteasen (Mucorpepsin; EC 3.4.23.23) gewonnen [2], die nahezu identisch und relativ thermostabil sind [3]. Darüber hinaus können Pepsin und Chymosin inzwischen auch mit Hilfe gentechnisch veränderter Mikroorganismen produziert werden.

Da es an den verschiedenen Arbeitsplätzen nicht selten zu einer gleichzeitigen Exposition gegenüber Pepsin und Chymosin bzw. mikrobiellen Labersatzstoffen kommt, soll die entsprechende Literatur gemeinsam ausgewertet werden.

\section{Literaturauswertung}

$\nabla$

Mit Hilfe geeigneter Stichwörter in sinnvoller Kombination wurden Veröffentlichungen in der Datenbank „PubMed“ recherchiert. Im Original zugängliche relevante Publikationen über Studien zur sensibilisierenden Wirkung von Pepsin, Chymosin und mikrobiellen Labersatzstoffen wurden hinsichtlich der klinischen Befunde ausgewertet. Erfasst wurden neben der Zahl der exponierten Personen die Prävalenzen der einzelnen Symptome bzw. Diagnosen. Darüber hinaus wurde festgehalten, mit welchen Methoden (Hauttest, Bestimmung spezifischer IgE-Antikörper, Provokationstest) und unter Verwendung welcher Testlösungen eine Sensibilisierung diagnostiziert wurde.

\section{Ergebnisse \\ $\nabla$}

Die Auswertung der Literatur zeigt, dass Pepsin, Chymosin und mikrobiellen Labersatzstoffen ebenso wie auch anderen Enzymen ( $\alpha$-Amylase [4], Subtilisin [5], Cellulase [6] und Xylanase [7], Papain [8], Bromelain [9], Phytasen [10]) nach beruflicher Exposition eine atemwegssensibilisierende Wirkung zukommen kann. Hautsensibilisierende Wirkungen wurden in der Literatur bisher nicht beschrieben. Einen zusammenfassenden Überblick über die Ergebnisse der Literaturauswertung sowie nähere Angaben zu den durchgeführten Tests gibt $\bullet$ Tab. 1.

\section{Atemwegssensibilisierende Wirkung $\nabla$}

Bei einem 30-jährigen Mann kam es ca. ein Jahr nach Beginn der Tätigkeit in der Qualitätskontrolle eines pharmazeutischen Unternehmens zur Verschlechterung seiner seit Teenagerzeit bestehenden allergischen Beschwerden. Ursprünglich waren die Beschwerden, die auf eine Sensibilisierung gegen Pollen und Katze zurückzuführen waren, medikamentös gut kontrollierbar. Seit Beginn der Tätigkeit nahmen nun sowohl die rhinokonjunktivalen als auch die asthmatischen Beschwerden zu und wiesen einen klaren Arbeitsbezug auf. Insbesondere wenn in dem Betrieb Pepsin, Kräuter oder Pollenextrakte verarbeitet wurden, litt der Patient unter starken asthmatischen Beschwerden. Dabei handelte es sich um natürliches Pepsin aus Schweine- und Rindermägen. Peak-Flow-Messungen am Arbeitsplatz und zu Hause zeigten deutliche Schwankungen in der PEFR (peak expiratory flow rate) mit den niedrigsten Werten (ca. 75\% vom Ausgangswert) während der Arbeitsperioden. Der Haut-Pricktest mit dem Pepsinpulver vom Arbeitsplatz des Patienten verlief positiv; bei 9 von 10 atopischen Kontrollpersonen hingegen negativ. Lediglich bei einem Kontrollprobanden kam es zu einer kleinen Quaddel ( $3 \mathrm{~mm})$. Zusätzlich wurde bei dem Patienten ein kommerzielles Pepsinprodukt im Pricktest eingesetzt und auch dieser Test verlief positiv. Im unspezifischen Provokationstest mit Histamin reagierte der Patient hyperreagibel. Im Rahmen eines arbeitsplatzbezogenen Inhalationstestes (AIT) musste der Proband das Pepsinpulver von seinem Arbeitsplatz so handhaben wie bei der am Tag zuvor negativ verlaufenen Kontrolltestung das Laktosepulver. Bereits nach einer Minute kam es zunächst zu Rhinokonjunktivitis und Atembeschwerden und schließlich zu einem Abfall der Einsekundenkapazität $\left(\mathrm{FEV}_{1}\right)$ um $69 \%$, dem sofort medikamentös entgegengewirkt wurde. Mittels RAST (Radio Allergo Sorbent Test) konnte ein erhöhtes spezifisches IgE (sIgE) gegen Pepsin 
Tab. 1 Klinische Untersuchungen zur atemwegssensibilisierenden Wirkung von Pepsin, Chymosin und mikrobiellen Labersatzstoffen beim Menschen

\begin{tabular}{|c|c|c|c|c|c|c|}
\hline \multirow{2}{*}{$\begin{array}{l}\text { Exposition } \\
\text { gegen }\end{array}$} & \multirow{2}{*}{$\begin{array}{l}\text { Zahl der } \\
\text { Untersuchten }\end{array}$} & \multirow{2}{*}{$\begin{array}{l}\text { arbeitsplatz- } \\
\text { bezogene } \\
\text { Symptome }\end{array}$} & \multicolumn{3}{|l|}{ Nachweismethode } & \multirow[t]{2}{*}{ Literatur } \\
\hline & & & Pricktest & $\begin{array}{l}\text { spezifische } \\
\text { IgE-Antikörper }\end{array}$ & $\begin{array}{l}\text { bronchiale } \\
\text { Provokation }\end{array}$ & \\
\hline Pepsin & 1 & $\begin{array}{l}\text { Rhinitis, Konjunkti- } \\
\text { vitis, Atemwegs- } \\
\text { beschwerden }\end{array}$ & positiv (Pepsin, $10 \mathrm{mg} / \mathrm{ml}$ ) & $\begin{array}{l}\text { RAST } \\
\text { Pepsin-slgE } \uparrow \text { (im Ver- } \\
\text { gleich zu } 11 \text { atopischen } \\
\text { Kontrollen) }\end{array}$ & $\begin{array}{l}\text { positiv mit Pepsin-Pulver } \\
\text { nach } 1 \text { min.: } \\
\text { FEV }_{1} \text {-Abfall } 69 \%\end{array}$ & [11] \\
\hline Pepsin & 1 & Husten, Asthma & 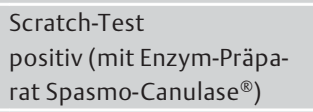 & $\begin{array}{l}\text { RAST } \\
\text { positiv (Pepsin) }\end{array}$ & n.d. & [12] \\
\hline $\begin{array}{l}\text { Pepsin, } \\
\text { Chymosin }\end{array}$ & 1 & $\begin{array}{l}\text { Rhinits, Konjunkti- } \\
\text { vitis, Husten, Atem- } \\
\text { wegsbeschwerden }\end{array}$ & $\begin{array}{l}\text { positiv (Pepsin, } 1 \mathrm{mg} / \mathrm{ml} \text { ) } \\
\text { negativ (Chymosin, } 100 \\
\mathrm{mg} / \mathrm{ml} \text { ) }\end{array}$ & $\begin{array}{l}\text { RAST } \\
\text { negativ } \\
\text { (Pepsin u. Chymosin) }\end{array}$ & $\begin{array}{l}\text { positiv mit Pepsin-Pulver } \\
\text { FEV }_{1} \text {-Abfall nach } 1 \text { min.: } \\
41 \% \\
\text { nach } 3 \text { h: } 29 \% \\
\text { negativ mit Chymosin- } \\
\text { Pulver }\end{array}$ & [13] \\
\hline Pepsin & 1 & $\begin{array}{l}\text { Atemwegsbeschwer- } \\
\text { den (progredienter } \\
\text { Verlauf trotz Tätig- } \\
\text { keitsaufgabe) }\end{array}$ & positiv (Pepsin, $1 \%$ ig) & $\begin{array}{l}\text { k.A. } \\
\text { positiv (Pepsin, 4,2 kU/I) }\end{array}$ & $\begin{array}{l}\text { positiv mit Pepsin-Pulver } \\
\text { Atemnot und Anstieg } \\
\text { Atemwegswiderstand } \\
\text { v. } 0,2 \text { auf } 1,0 \mathrm{kPa} / \mathrm{l} / \mathrm{s}\end{array}$ & [14] \\
\hline Pepsin & 1 & Rhinits, Konjunktivitis & positiv (Pepsin, $10 \mathrm{mg} / \mathrm{ml}$ ) & $\begin{array}{l}\text { k.A. } \\
\text { positiv (Pepsin, 5,6 kU/l) }\end{array}$ & $\begin{array}{l}\text { positiv }(10 \mathrm{mg} / \mathrm{ml} \text {, } \\
\text { konjunktival) }\end{array}$ & [15] \\
\hline $\begin{array}{l}\text { Pepsin, Chy- } \\
\text { mosin und } \\
\text { mikrobielle } \\
\text { Labersatz- } \\
\text { stoffe }\end{array}$ & 35 & $\begin{array}{l}\text { 24/35 Atemwegs- } \\
\text { beschwerden, } \\
\text { 21/35 Rhinitis, } \\
\text { Konjunktivitis }\end{array}$ & $\begin{array}{l}\text { positiv mit Pepsin 12/35 } \\
\text { Chymosin: 10/35 } \\
\text { Mucorpepsin: 10/35 } \\
\text { Endothiapepsin: } 2 / 35 \\
(200 \mathrm{IMCU} / \mathrm{ml})\end{array}$ & $\begin{array}{l}43 \% \text { der Pricktest- } \\
\text { Positiven: erhöhtes } \\
\text { Gesamt-IgE } \\
\text { (MW: } 322 \mathrm{IU} / \mathrm{ml}, \text { Max. } \\
505 \mathrm{IU} / \mathrm{ml})\end{array}$ & n.d. & [17] \\
\hline $\begin{array}{l}\text { Endothia- } \\
\text { pepsin }\end{array}$ & 1 & Rhinitis, Asthma & $\begin{array}{l}\text { positiv (Endothiapepsin } \\
1: 100.000 ; \text { Scratch mit } \\
\text { Endothiapepsin-Pulver) }\end{array}$ & n.d. & n.d. & [1] \\
\hline Mucorpepsin & 1 & $\begin{array}{l}\text { Husten, Asthma, } \\
\text { Hautbeschwerden }\end{array}$ & $\begin{array}{l}\text { positiv } \\
\text { (Mucorpepsin, 0,01\%; } \\
\text { Molke) }\end{array}$ & n.d. & $\begin{array}{l}\text { positiv (Mischen v. } 15 \mathrm{ml} \\
\text { Ferment mit } 350 \mathrm{ml} \mathrm{H}_{2} 0 \text { ) } \\
\text { FEV }_{1} \text {-Abfall } \\
\text { sofort: } 20 \% \\
\text { nach } 7 \mathrm{h:} 31 \%\end{array}$ & [19] \\
\hline
\end{tabular}

n. d.: nicht durchgeführt, k. A.: keine Angaben, IMCU: International Milk Clotting Units

im Vergleich zu elf atopischen Kontrollen nachgewiesen werden [11].

Ein 34-jähriger Krankenpfleger litt zunächst gehäuft unter trockenem Reizhusten und danach unter wiederholten Asthmaanfällen, zunächst ohne erkennbaren Grund. Die allergologische Abklärung auf ubiquitäre Allergene sowie Nahrungsmittel verlief negativ. Mit $100 \mathrm{E} / \mathrm{ml}$ ergab sich allerdings ein grenzwertiger Befund für Gesamt-IgE. Es zeigte sich, dass die Symptome im $\mathrm{Zu}-$ sammenhang mit dem Zerdrücken verschiedener Medikamente für einen Patienten mit Magensonde standen. Im Scratch-Test ergab das enzymhaltige Präparat Spasmo-Canulase ${ }^{\circledR}$ eine stark positive Reaktion. Mittels RAST konnte sIgE gegen Trypsin vom Schwein, nicht jedoch gegen Rinder-Trypsin nachgewiesen werden. Zudem war sIgE gegen Pepsin geringfügig, aber signifikant, erhöht [12].

Ein 39-jähriger nicht-atopischer Mann war bereits seit acht Jahren als Lagerist in einer Käserei beschäftigt, bevor er in die Produktion wechselte und dort Kontakt zu Milch und verschiedenen Enzymen, u.a. auch Pepsin und Chymosin, hatte. Etwa ein Jahr nach diesem Tätigkeitswechsel traten bei ihm erstmals Rhinokonjunktivitis, Husten und Atemnot auf. Die Symptome begannen typischerweise kurz nach Arbeitsbeginn und hielten die Woche über an, besserten sich am Wochenende und gingen während des Urlaubs nahezu komplett zurück. Peak-Flow-Messungen bei fünftägiger Abwesenheit von der Arbeit zeigten keinerlei
Auffälligkeiten, am ersten Arbeitstag kam es hingegen nach 30 Minuten zu rhinokonjunktivalen und asthmatischen Beschwerden und zu einem 37\%igen Abfall der PEFR vom Ausgangswert. Im unspezifischen Provokationstest mit Methacholin reagierte der Patient positiv, während er im Haut-Pricktest auf keines der getesteten ubiquitären Allergene reagierte. Auch die Pricktestung mit nativer Kuhmilch, Flüssigferment, Calcium sowie Chymosin verlief negativ, allerdings kam es zu einer deutlichen Reaktion auf Pepsin und auf Lysozym. Drei atopische und zwei nicht-atopische Kontrollpersonen zeigten keine Reaktion. Im AIT mit trockenem Pepsinpulver entwickelte der Patient bereits nach einer Minute Rhinokonjunktivitis, Husten, Atemnot und ein Glottisödem. Dabei kam es zu einem $\mathrm{FEV}_{1}$-Abfall um $41 \%$, der medikamentös behandelt wurde; drei Stunden später folgte ein erneuter $\mathrm{FEV}_{1}$-Abfall um 29\%. Ebenfalls positiv verlief der AIT mit Lysozym, negativ jedoch der mit Chymosin. Mittels RAST konnte lediglich sIgE gegen Lysozym, nicht jedoch gegen Pepsin und Chymosin nachgewiesen werden [13].

Eine 56-jährige Frau war seit 19 Jahren in der Trichinenuntersuchung beschäftigt und führte dort seit elf Jahren Testungen nach der Digestionsmethode durch, bei der sie regelmäßig mit Pepsin in Kontakt kam. Bereits ein Jahr nach Aufnahme dieser Tätigkeit traten am Arbeitsplatz erstmals asthmatische Beschwerden auf. Zeitgleich entwickelte die Patientin eine Rhinitis allergica mit Beschwerden von Februar bis April. Ein Haut-Pricktest mit wässri- 
ger Pepsinlösung verlief positiv, bei drei nicht-exponierten Kontrollpersonen hingegen negativ. Am Ende des 10-minütigen AITs, bei dem die Patientin Pepsinpulver von einem Gefäß in ein anderes schüttete, kam es zu Rhinokonjunktivitis und Luftnot. Der Atemwegswiderstand (Rtot) stieg dabei von ursprünglich 0,2 auf knapp über $1 \mathrm{kPa} / \mathrm{l} / \mathrm{s}$ an. Die Bestimmung des slgE ergab einen Wert von 4,2 kU/l für Pepsin. Wie zwei Jahre nach der ersten Untersuchung festgestellt wurde, war die Atemwegserkrankung trotz permanenter Abwesenheit vom Arbeitsplatz progredient verlaufen, und eine Dauermedikation mit Kortikosteroiden und $\beta$-Mimetika war zwischenzeitlich erforderlich geworden [14]. Ebenfalls mit der Trichinenuntersuchung betraut war eine 22jährige Schlachthof-Beschäftigte, die sieben Monate nach Tätigkeitsaufnahme rhinokonjunktivale Beschwerden entwickelte. Der Haut-Pricktest mit Pepsin verlief bei der Patientin positiv, bei mehreren atopischen bzw. nicht-atopischen Kontrollen jedoch negativ. Der konjunktivale Provokationstest mit Pepsin führte bei der Patientin zu einem konjunktivalen Erythem mit Tränenfluss und Juckreiz, blieb bei drei atopischen sowie drei nicht-atopischen Kontrollen jedoch folgenlos. Die Lungenfunktion der Patientin lag im Normbereich (forcierte Vitalkapazität (FVC): 86\% Soll, FEV 1 : 96\% Soll, FEV 1 /FVC: $91 \%$ ). Spezifisches IgE war nachweisbar gegen Pepsin, Hausstaubmilbe und gegen Rinderepithelien. Nach gelelektrophoretischer Auftrennung der Pepsinlösung wurde durch Inkubation mit dem Serum der Patientin im Immunoblot eine sIgE-bindende 43 kDa-Bande erkennbar [15].

In einer weiteren Publikation, die jedoch in Russisch verfasst ist und für die deshalb nur das englischsprachige Abstrakt zur Verfügung steht, wird von einem Mann berichtet, der seit mehr als 30 Jahren in der Herstellung pulverförmigen Pepsins beschäftigt war und Hinweise auf eine beruflich bedingte exogen-allergische Alveolitis zeigte [16].

In einer Firma, in der sowohl natürliches als auch synthetisches Labenzym hergestellt wurde, nahmen 35 von 38 Beschäftigten an einer medizinischen Untersuchung teil. Das Alter der Teilnehmer betrug im Mittel 40,6 Jahre (27-61 Jahre), die Beschäftigungsdauer lag zwischen 3 Monaten und 30 Jahren. Mittels eines Fragebogens wurden die Symptome der Beschäftigten erfasst: 24 der 35 Befragten gaben asthmatische Beschwerden an, 12 (34\%) davon mit Arbeitsplatzbezug. Rhinokonjunktivitis gaben 21 an, bei zehn (29\%) Beschäftigten verschlimmerten sich die Symptome am Arbeitsplatz. Für den Haut-Pricktest wurden verschiedene Enzymlösungen hergestellt, die mit NaCl-Lösung auf eine Enzymaktivität von $200 \mathrm{IMCU} / \mathrm{ml}$ (International Milk Clotting Units/ml) eingestellt wurden. Quaddeln von $3 \mathrm{~mm}$ oder größer galten als positiv. Auf mindestens ein Labenzym reagierten im Pricktest 14 (40\%) der 35 Beschäftigten positiv. Dabei reagierten auf natürliches Pepsin aus Rindermägen bzw. Chymosin aus Kälbermägen 12 (34\%) bzw. zehn (29\%) positiv. Auf Mucorpepsin aus Rhizomucor miehei sowie auf Endothiapepsin aus Cryphonectria reagierten zehn (29\%) bzw. zwei (6\%) Patienten und auf ein höher gereinigtes Chymosin aus Aspergillus niger-Kulturen vier (11\%) der 35 Untersuchten. Von 28 nicht-exponierten Kontrollpersonen reagierte lediglich eine auf eines der fünf Labenzyme. Sechs der 14 Beschäftigten, die im Hauttest auf mindestens eines der Labenzyme reagierten, wiesen ein erhöhtes Gesamt-IgE auf. Insgesamt zeigten die Personen, die Umgang mit pulverförmigen Labenzymen hatten, häufiger eine Sensibilisierung (3 von 4) als diejenigen, die Umgang mit flüssigen Enzymen hatten (4 von 8) [17]. Bereits 1976 wurde bei einem 45-jährigen Käsermeister eine allergische Reaktion auf das Labersatzferment Endothiapepsin beschrieben. Der Patient litt seit Beginn der Verwendung des Labferments vier Jahre zuvor zunächst unter berufsbedingter Rhinitis, später unter asthmoider Bronchitis und Auswurf und schließlich unter Asthma. Die spirometrische Untersuchung ergab eine deutliche obstruktive Ventilationsstörung mit reversibler Komponente. Während der Intrakutantest mit Umweltallergenen und Schimmelpilzen negativ verlief, ergab der Scratchtest mit Endothiapepsin-Pulver ein dreifach positives Ergebnis. Im Gegensatz dazu verlief der Scratchtest bei drei Kontrollpersonen negativ. Die Schwellenwertbestimmung mit steriler Endothiapepsin-Lösung zur Erfassung des Sensibilisierungsgrades ergab im Pricktest eine einfach positive Reaktion bei einer Verdünnung von 1:100000. Die Präzipitinanalyse mit Endothiapepsin verlief negativ. Unter Einhaltung einer entsprechenden Expositionskarenz und bei Verwendung von tierischem Lab aus Kälbermagen verschwanden die Symptome des Beschäftigten [1].

Auch eine 38-jährige Käsermeisterin, die Umgang mit pulverförmigem Endothiapepsin hatte, klagte ein Jahr nach Tätigkeitsbeginn über berufsbezogene Atemwegsbeschwerden. Der Scratchtest am Rücken mit Endothiapepsin verlief positiv, bei zehn Kontrollpersonen jedoch negativ. Ebenfalls negativ war der Scratchtest mit einem natürlichen Labferment aus Kälbermagen. Nachdem in dem Betrieb Labferment in flüssiger anstatt in pulverförmiger Form verwendet wurde, war die Beschäftigte beschwerdefrei. Aufgrund dieser Kasuistik wurden 16 Beschäftigte von zwei Käsereien befragt und untersucht. Zwei Beschäftigte hatten Umgang mit einer flüssigen Labferment-Zubereitung aus Rhizomucor miehei und fünf mit pulverförmigem Labferment angegeben. Fünf Beschäftigte klagten über Rhinitis und Husten beim Umgang mit Labferment, drei berichteten über Bläschenbildung an den Händen und Unterarmen. Die Hautsymptome verliefen mild und bedurften keiner Behandlung. Alle 16 Beschäftigten wurden im Scratchtest mit beiden verwendeten sowie mit fünf weiteren Labferment-Zubereitungen getestet. Die Ergebnisse wurden nach 15 Minuten und $24 \mathrm{~h}$ abgelesen - alle Tests verliefen jedoch negativ [18].

Bei einer 38-jährigen Beschäftigten trat kurz nach Aufnahme der Tätigkeit in der Käseherstellung Husten, Atemnot und Giemen auf. Die Beschäftigte mixte 3 Liter einer flüssigen MucorpepsinZubereitung aus Rhizomucor miehei mit 7 Liter Wasser und versetzte 4000 Liter Milch mit diesem Gemisch. Spritzer der Labferment-Zubereitung führten zu urtikariellen Hautreaktionen. Trotz Behandlung mit Corticoid-Spray traten weiterhin arbeitsplatzbezogene Symptome auf, PEF-Messungen lieferten jedoch normale Befunde. Die Patientin wies eine mäßige unspezifische bronchiale Hyperreaktivität auf. Im Pricktest zeigten sich positive Reaktionen auf Mucorpepsin und auf die Molke. Bei einem bronchialen Provokationstest mischte die Patientin $15 \mathrm{ml}$ Ferment-Zubereitung in einer Schale mit $350 \mathrm{ml}$ kaltem Wasser und zeigte eine bronchiale Sofortreaktion mit 20\%igem Abfall des $\mathrm{FEV}_{1}$ sowie eine Spätreaktion mit Abfall des $\mathrm{FEV}_{1}$ um 31\% nach 7 Stunden [19].

\section{Schlussfolgerung \\ $\nabla$}

Durch eine Anzahl von Publikationen sind Fälle von spezifischer Überempfindlichkeit der Atemwege durch die Aspartatproteasen Pepsin und Chymosin sowie durch mikrobielle Labersatzstoffe belegt. Da es an den betreffenden Arbeitsplätzen häufig zu gleichzeitiger Exposition gegenüber den genannten Enzymen kommt und insbesondere die für die medizinischen Testungen 
verwendeten natürlichen Enzympräparate kaum rein zu gewinnen sind, erscheint eine Einzelbetrachtung der Lab-Enzyme nicht sinnvoll. Vielleicht stimmen auch aus diesen Gründen Symptome, Hauttestergebnisse, der Nachweis spezifischer IgE-Antikörper und die Ergebnisse spezifischer Provokationsteste nicht immer exakt überein. Insgesamt scheint jedoch ein immunologischer Wirkmechanismus gesichert.

\section{Interessenkonflikt}

$\nabla$

Die Autoren geben an, dass kein Interessenkonflikt besteht.

\section{Literatur}

1 Wüthrich B. Zum Allergenkatalog beruflicher Inhalationsallergien: Asthma bronchiale auf ein synthetisches Labferment, Rhinitis allergica auf Protease und Rhinoconjunctivitis allergica auf Puppe des Falters Galleria mellonella. Berufsdermatosen 1976; 24: 123-131

2 Baudys M, Foundling S, Pavlík M et al. Protein chemical characterization of Mucor pusillus aspartic proteinase. Amino acid sequence homology with the other aspartic proteinases, disulfide bond arrangement and site of carbohydrate attachment. FEBS Lett 1988; 235: 271 - 274

3 Yang J, Teplyakov A, Quail JW. Crystal structure of the aspartic proteinase from Rhizomucor miehei at 2.15 A resolution. J Mol Biol 1997; 268: $449-459$

4 Baur X, Chen Z, Sander I. Isolation and denomination of an important allergen in baking additives: alpha-amylase from Aspergillus oryzae (Asp o II). Clin Exp Allergy 1994; 24: 465-470

5 van Kampen $V$, Merget $R$. Berufliche Atemwegssensibilisierungen durch Subtilisine. Pneumologie 2002; 56: $182-186$

6 van Kampen $V$, Lessmann $H$, Brüning $T$ et al. Berufliche Allergien gegen Cellulasen. Pneumologie 2003; 57: 388-391
7 van Kampen $V$, Merget R, Brüning $T$. Berufliche Allergien gegen Xylanasen. Pneumologie 2004; 58: 103-106

8 van Kampen V, Merget R, Brüning T. Berufliche Allergien gegen Papain. Pneumologie 2005; 59: 405-410

9 van Kampen $V$, Merget $R$, Brüning T. Berufliche Allergien gegen Bromelain. Pneumologie 2007; 61: 159-161

10 van Kampen $V$, Merget $R$, Brüning $T$. Berufliche Allergien gegen Phytase. Pneumologie 2008; 62: 707-710

11 Cartier A, Malo JL, Pineau L et al. Occupational asthma due to pepsin. J Allergy Clin Immunol 1984; 73: 574-577

12 Hartmann AL, Wüthrich B, Baur X. Allergisches Asthma auf Enzyme in Arzneimitteln. Schweiz Med Wochenschr 1984; 114: 916-917

13 Añíbarro Bausela B, Fontela JL. Occupational asthma in a cheese worker. Allergy 1996; 51: 960-961

14 Drexler H, Beyer B. Berufsbedingtes allergisches Asthma einer Trichinenschauerin durch Pepsinpuder. Arbeitsmed Sozialmed Umweltmed 1997; 32: $145-147$

15 Marquès LI, Lara S, Abós T et al. Occupational rhinitis due to pepsin. J Investig Allergol Clin Immunol 2006; 16: 136 - 137

16 Krasniuk EP, Petrova IS, Pilinskii VV. Exogenous allergic alveolitis in workers engaged in the manufacture of pepsin. Lik Sprava 2001: $168-171$ (in Russisch)

17 Jensen A, Dahl S, Sherson $D$ et al. Respiratory complaints and high sensitization rate at a rennet-producing plant. Am J Ind Med 2006; 49: $858-861$

18 Niinimăki A, Saari S. Dermatological and allergic hazards of cheesemakers. Scand J Work Environ Health 1978; 4: 262-263

19 Suojalehto H, Tuppurainen M, Lindstrom I, Nordman H. Occupational asthma due to milk coagulant protease. Poster-Abstract P3845. European Respiratory Society Annual Congress, Berlin, 7.Oktober 2008 http://www.ersnet.org/learning_resources_player/abstract_print_08/ files/376.pdf 